Check for updates

Cite this: Chem. Sci., 2019, 10, 2396

๑ All publication charges for this article have been paid for by the Royal Society of Chemistry

Received 20th September 2018

Accepted 2nd January 2019

DOI: $10.1039 / \mathrm{c} 8 \mathrm{sc} 04199 \mathrm{~g}$

rsc.li/chemical-science

\section{Charge transfer complexation boosts molecular conductance through Fermi level pinning $\dagger$}

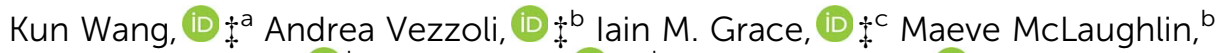 \\ Richard J. Nichols, (D) ${ }^{\mathrm{b}}$ Bingqian Xu, (D) ${ }^{* a d}$ Colin J. Lambert (D) ${ }^{* c}$ \\ and Simon J. Higgins (D) *b
}

\begin{abstract}
Interference features in the transmission spectra can dominate charge transport in metal-molecule-metal junctions when they occur close to the contact Fermi energy $\left(E_{\mathrm{F}}\right)$. Here, we show that by forming a chargetransfer complex with tetracyanoethylene (TCNE) we can introduce new constructive interference features in the transmission profile of electron-rich, thiophene-based molecular wires that almost coincide with $E_{\mathrm{F}}$. Complexation can result in a large enhancement of junction conductance, with very efficient charge transport even at relatively large molecular lengths. For instance, we report a conductance of $10^{-3} \mathrm{G}_{0}$ ( $\sim 78 \mathrm{nS}$ ) for the $\sim 2 \mathrm{~nm}$ long $\alpha$-quaterthiophene:TCNE complex, almost two orders of magnitude higher than the conductance of the bare molecular wire. As the conductance of the complexes is remarkably independent of features such as the molecular backbone and the nature of the contacts to the electrodes, our results strongly suggest that the interference features are consistently pinned near to the Fermi energy of the metallic leads. Theoretical studies indicate that the semi-occupied nature of the charge-transfer orbital is not only important in giving rise to the latter effect, but also could result in spin-dependent transport for the charge-transfer complexes. These results therefore present a simple yet effective way to increase charge transport efficiency in long and poorly conductive molecular wires, with important repercussions in single-entity thermoelectronics and spintronics.
\end{abstract}

\section{Introduction}

The ability to manipulate quantum transport through single molecules is key to building functional molecular electronic devices. Towards this goal, various approaches have been explored in metal-molecule-metal junctions to exert control on molecular conductance, including optical illumination, ${ }^{1-3}$ gating the electrochemical ${ }^{4-7}$ or electrostatic ${ }^{8-11}$ environment, and through reversible supramolecular interactions such as solvent effects, ${ }^{\mathbf{1 2 - 1 6}}$ complexation, ${ }^{\mathbf{1 7 - 2 4}} \pi$-stacking interactions ${ }^{25-31}$ and hydrogen-bonding. ${ }^{32-34}$ Another way that has been proposed to chemically control the charge-transport properties of a molecular junction is by harnessing quantum interference

${ }^{a}$ Department of Physics and Astronomy \& NanoSEC, University of Georgia, 220 Riverbend Road, Athens, GA 30602, USA. E-mail: bxu@engr.uga.edu

${ }^{b}$ Department of Chemistry, University of Liverpool, Crown Street, Liverpool L69 7ZD, UK. E-mail: shiggins@liverpool.ac.uk

${ }^{c}$ Department of Physics, Lancaster University, Lancaster LA1 4YB, UK. E-mail: $c$. lambert@lancaster.ac.uk

${ }^{d}$ College of Engineering \& NanoSEC, University of Georgia, 220 Riverbend Road, Athens, GA 30602, USA

$\dagger$ Electronic supplementary information (ESI) available: Synthetic procedures and characterisation transcripts for the compounds used in this study. Additional single-molecule conductance data and further analysis. Details on the theoretical calculations. See DOI: 10.1039/c8sc04199g

\$ These authors contributed equally to this work. phenomena with energy close to the electrode Fermi level $E_{\mathrm{F}}$. Drawing inspiration from the seminal work of Sautet and Joachim, ${ }^{35}$ destructive interferences have been introduced, for instance, by exploiting meta-connectivity in $\pi$-conjugated systems, ${ }^{36-39}$ cross-conjugation, ${ }^{40-42}$ or the unique $\sigma$-bonding properties of organosilanes, ${ }^{43}$ and these have been employed to lower molecular conductance for the fabrication of efficient molecular insulators. In addition to providing a way to control molecular conductance, the presence of such transport features also results in higher Seebeck coefficients (a measure of thermoelectric conversion) at room temperature, ${ }^{44}$ which is a highly sought-after and promising property of molecular nanodevices. The conductance decrease associated with destructive interferences, however, reduces the power factor and figure of merit of these devices, thereby limiting their suitability for functional devices. Constructive interference in quantum transport have been well known in the physics literature, since the classic paper of Breit and Wigner, ${ }^{45}$ and transmission resonances in 1dimensional systems have been widely studied both in the presence of many scatterers ${ }^{4-48}$ and in the case of a single scatterer. ${ }^{35}$ Conductance in multi-path structures was examined from a physical ${ }^{\mathbf{4 9 , 5 0}}$ and chemical perspective. ${ }^{51}$ The latter suggested that when two conducting paths are connected in parallel within a molecule, the resulting conductance should be increased by a factor of four, rather than the factor of two 
observed in classical electrical networks. The prediction was later experimentally tested ${ }^{52,53}$ by measuring the conductance of analogous pairs of double-backbone and single-backbone molecular junctions, and their conductance ratio was found to lie between 3.3 and 1.6 (i.e. ratios were either higher or lower than the classical value of two, but did not reach the predicted ratio of four). Reliable experimental strategies for the introduction of constructive quantum interference features resulting in large conductance boosts are therefore lacking or inefficient. As a consequence, the development of robust methods to substantially increase conductance through constructive interference would provide valuable strategies for the design of molecular systems for efficient charge transport and thermoelectric conversion.

Among the supramolecular interactions mentioned earlier, charge-transfer (CT) complexation is of particular interest from the point of view of electronic devices. Several ground-breaking discoveries, such as the first organic material with metallic behaviour (TTF:TCNQ) $)^{54}$ and the first organic superconductor (Bechgaard salt, [TMTSF $\left.]_{2} \mathrm{PF}_{6}\right)^{55}$ used CT complexes as bulk conductors, and complexation in nanoscale junctions has been used to gate molecular conductance. ${ }^{17}$ We further explored the application of CT complexes in single-molecule electronics and we report here a general approach to the introduction of constructive quantum interference that can be applied to conventional, fully conjugated, molecular wires. CT complexation introduces additional resonances in their transmission profile, and these resonances have a Fano line-shape (as opposed to the more common Breit-Wigner distribution) characterised by an antiresonance followed by a sharp positive contribution. As these features arise from a part-filled orbital, their energetic position is predicted by simple band theory to be always near to the Fermi level of the electrodes $E_{\mathrm{F}}$. The presence of a transport feature near $E_{\mathrm{F}}$ in the transmission profile is of the utmost importance for the development of both long-range tunnelling and highly efficient thermoelectric power conversion at room temperature, and the ability to reliably pin it at the desired energy level is also a very sought-after property in molecular wire design. We demonstrate empirically that the Fano resonance introduced by the formation of a chargetransfer complex of a molecular wire with ethenetetracarbonitrile (TCNE or "tetracyanoethylene") is reliably placed at energy values close to the Fermi level of the electrodes ("pinning"), which results in complexes having conductance almost independent of the nature of the molecular backbone and contacts to the electrodes. The pinning effect results in a greatly reduced attenuation coefficient $\beta$ of the complexes across an oligomeric thienyl series, yielding efficient charge transport and large conductance values even through relatively long molecular wires.

\section{Results and discussion}

We focussed our study on a series of oligothiophenes, terminated at each end with methyl thioether contacts. Oligothiophenes were chosen as electron-rich heteroaryl units, which are known to form 1:1 CT complexes with strong electron acceptors such as TCNE. ${ }^{56-58}$ The compounds used in this study are presented in Fig. 1a, and details of the synthetic procedures can be found in the ESI. $\dagger$

We started our investigation by assessing the physical properties of the charge transfer complexes of these oligothiophene-based molecular wires, in order to determine their suitability for single-molecule conductance studies. As shown in Fig. 2, UV-vis spectroscopy in $\mathrm{CH}_{2} \mathrm{Cl}_{2}$ shows that an absorption band in the $550-800 \mathrm{~nm}$ region, corresponding to the transition, arises upon complexation, confirming the formation of the charge-transfer complex.

Having established the fact that these thiomethylterminated oligothiophenes form CT complexes with TCNE, the scanning tunnelling microscopy-break junction (STM-BJ) technique ${ }^{59}$ was then used to fabricate and measure the electrical properties of molecular junctions. In brief, a Au STM tip was brought into contact with a $\mathrm{Au}(111)$ substrate, in the presence of either the sample molecule or its TCNE complex (prepared as described in the Methods section), as a preadsorbed sub-monolayer. The tip was then retracted while the tunnelling current was monitored. As the tip is crashed into the substrate, a fresh junction is formed, and on retraction this thins down to a single atom (point contact, characterised by its conductance $G_{0} \approx 77.48 \mu \mathrm{S}$ ), which is finally broken upon further withdrawal. When the point contact breaks, if a molecule (or molecules) binds to both Au contacts, then subsequent additional plateaux are seen in the current-distance plot at values much smaller than $G_{0}$, corresponding to charge transport through the molecular bridge. Eventually, as retraction

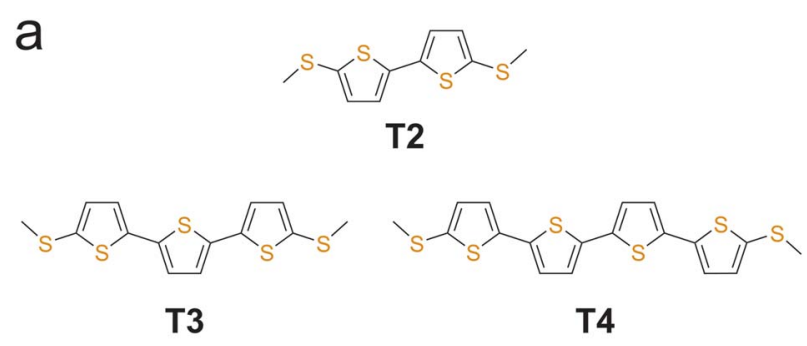

b
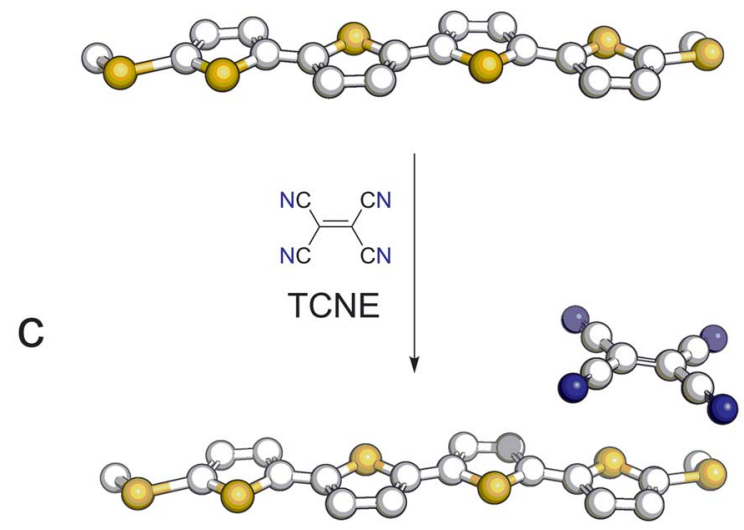

Fig. 1 (a) Structures and naming of the compounds used in this study. Representation of a molecular wire (b) and its TCNE complex (c). 

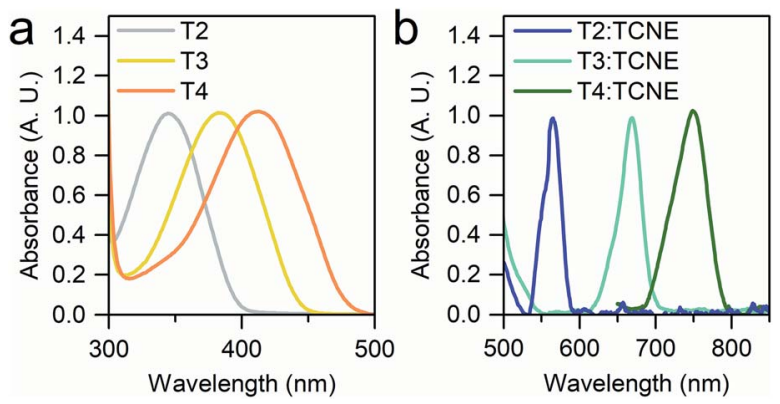

Fig. 2 UV-vis spectra of the T2-T4 series (a) and of their TCNE complexes (b) in $\mathrm{CH}_{2} \mathrm{Cl}_{2}$. A $1: 100$ ratio of molecule : TCNE was used to obtain (b). All the spectra are normalised, and the onset of the T4 $\pi$ $\rightarrow \pi^{*}$ band below $650 \mathrm{~nm}$ in (b) was removed for clarity. The CT bands are two orders of magnitude less intense than the $\pi \rightarrow \pi^{*}$ transition.

continues, the molecular junction breaks and the current abruptly falls to a very low value consistent with direct electrode tunnelling. Thousands of such traces are recorded for each set of experimental conditions, and the traces are compiled into conductance histograms. The plateaux result in peaks in the histogram at values below $G_{0}$, which represent the distribution of conductance values of the molecular junction, and the same traces are used for further plateau length analysis. More details can be found in the Methods section and in the ESI, $\uparrow$ and the conductance measurements are presented in Fig. 3.

The non-complexed oligothiophene series showed, as expected, an exponential decay of the conductance with increasing molecular length, with an associated decay constant $\beta=5.0 \mathrm{~nm}^{-1}$, in reasonable agreement with earlier data reported on the same system $\left(4 \mathrm{~nm}^{-1}\right) .^{60}$ The small differences in the absolute conductance values and in $\beta$ can be attributed to the different media used for the STM-BJ measurements, which is air in this study and 1,2,4-trichlorobenzene in Capozzi et al. It has already been demonstrated that the junction behaviour can be greatly influenced by the solvent used during the measurements..$^{12,14-16,61}$ The conductance decay upon exposure to TCNE, on the other hand, showed a greatly reduced attenuation factor $\beta$ of $1.7 \mathrm{~nm}^{-1}$, highlighting the almost length-independent conductance of the charge-transfer complexes, which lies relatively constant at approximately $10^{-3} G_{0}$. This allows efficient charge transport even at large molecular lengths $\left(10^{-3} G_{0}\right.$ conductance through a $\sim 2 \mathrm{~nm}$ molecular wire). Furthermore, the conductance boost upon complexation (as $G_{\text {COMPLEX }} / G_{\text {MOL }}$ ) follows an exponential increase as predicted. A summary of all conductance values can be found in Table 1 .

To rule out the possibility that the source of the high conductance feature consistently near $\sim 10^{-3} G_{0}$ is from TCNE itself, conductance measurements on samples either with no molecule or solely with TCNE were therefore performed as a control. No significant feature was found in the conductance histograms within the range of $10^{-5}$ to $1 G_{0}$ (see ESI, Fig. S3†). In addition, plateau length analysis revealed that the molecule:TCNE junctions have break-off distances similar to those of the corresponding uncomplexed molecular wires (see ESI, Fig. S2 $\dagger$ ), further excluding the contribution of TCNE alone. We thus
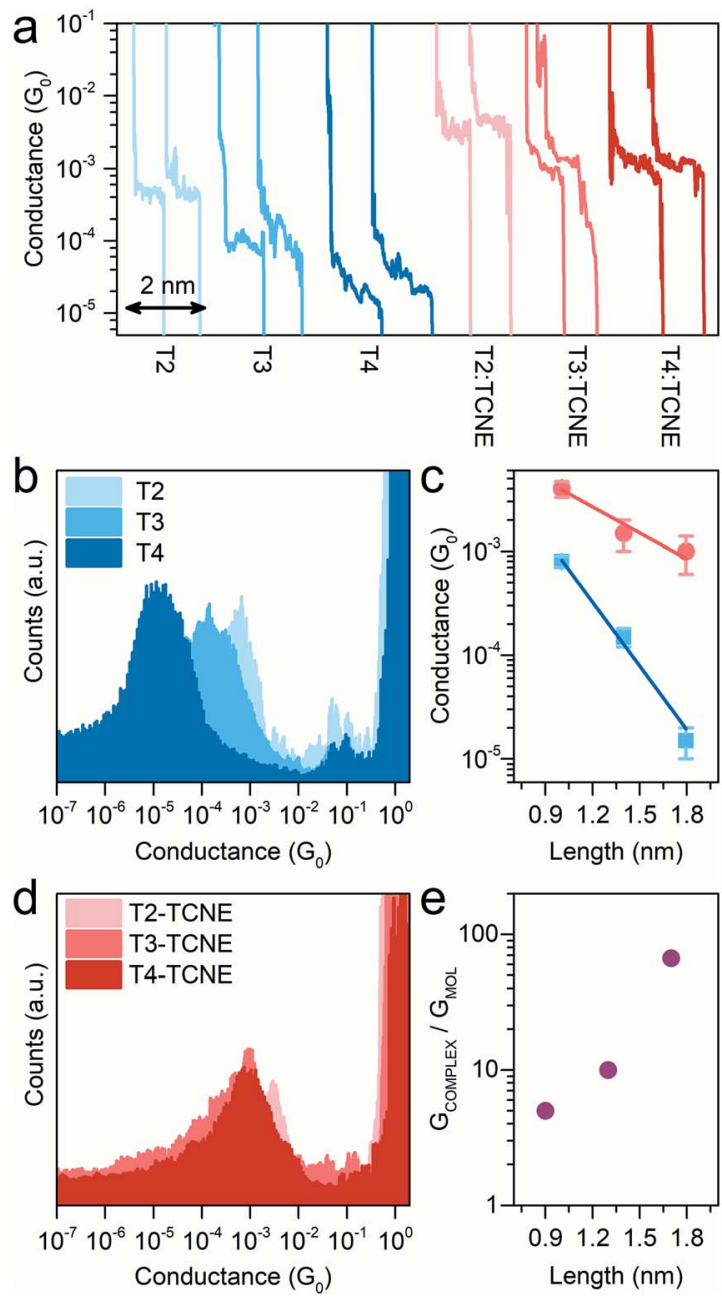

Fig. 3 Length-dependent conductance measurements of methylthioether terminated oligothiophenes with 2 (T2), 3 (T3) and 4 (T4) thiophene units. Example conductance vs. electrode displacement traces (a) of the isolated molecules (blue shades - from left to right: T2, T3 and T4) and the corresponding complexes with TCNE (red shades from left to right: T2:TCNE, T3:TCNE and T4:TCNE). Conductance histograms of T2, T3 and T4 (b) and their TCNE complexes (d). Conductance vs. molecular length plot (c) for the isolated molecules (blue) and the respective complexes with TCNE (red) and conductance enhancement vs. molecular length plot (e). Error bars in (c) represent the standard deviation. Molecular length calculated at DFT level (see Methods).

conclude that the conductance peak at $\sim 10^{-3} G_{0}$ observed from all molecule:TCNE samples is mainly contributed by the formation of molecule:TCNE complexes due to CT complexation.

To understand the observed phenomena, the junction behaviour was then modelled with DFT, focussing on a dynamics study of the geometrical fluctuations of the molecular wire trapped in the junction and of its TCNE complex. The zero-bias transmission coefficient $T(\mathrm{E})$ was obtained for 500 different configurations, sampling a range of structures of the CT complex in the junction, and the average finite-bias roomtemperature conductance was then calculated (details in the Methods section and examples of structures and relative density 
Table 1 Summary of single-molecule conductance values of the compounds presented in this study and their charge-transfer complexes with TCNE. Theoretical values are presented in brackets

\begin{tabular}{llll}
\hline Compound & Molecular conductance $\left(G_{0}\right)$ & TCNE complex conductance $\left(G_{0}\right)$ & Ratio: $G_{\text {COMPLEX }} / G_{\text {MOL }}$ \\
\hline T2 & $8 \times 10^{-4}\left(3 \times 10^{-3}\right)$ & $4 \times 10^{-3}\left(8 \times 10^{-3}\right)$ & $\sim 5(2)$ \\
T3 & $1.5 \times 10^{-4}\left(4 \times 10^{-4}\right)$ & $1.5 \times 10^{-3}\left(2 \times 10^{-3}\right)$ & $\sim 10(5)$ \\
T4 & $1.5 \times 10^{-5}\left(3 \times 10^{-5}\right)$ & $1 \times 10^{-3}\left(1 \times 10^{-3}\right)$ & $\sim 67(33)$
\end{tabular}

of states plots in the ESI $\dagger$ ). This approach was previously used ${ }^{17}$ to capture a realistic picture of the complexed molecular junction at room temperature, accounting for the fluctuations in the TCNE orientation and position with respect to the oligothiophene backbone. Spectroscopic and crystallographic data on CT complexes of oligothiophenes consistently report a $1: 1$ complex ratio, ${ }^{57,62,63}$ and this was used in our calculations. An additional Fano resonance ${ }^{64}$ has been previously observed in the DFT-calculated transmission curves for the TCNE complex of a bis(thialkyl)terthiophene ${ }^{17}$ which accounted for the conductance boost phenomenon, and similar resonances arise with TCNE complexation of the oligomeric series T2-T4. In the CT complexes, the donation of partial electron density to TCNE results in part-filled orbitals on the molecular backbone and TCNE itself, the latter acting as a scattering centre and generating the Fano resonance through quantum interference and accounting for the observed conductance change. The asymmetric line shape of Fano resonances ${ }^{36,64}$ occurs when a backbone orbital coupled to a continuum of states in the electrodes interacts with a pendant orbital, which in our case resides on the TCNE.

The sharp resonances in the individual transmission curves result in "bumps" in the thermally-averaged finite-bias calculated conductance, very near to $E_{\mathrm{F}}$ in energy (Fig. 4 ), which are responsible for the observed increase in conductance upon complexation. Calculations predict a $\beta$ value for the "naked" molecular wires of $6.5 \mathrm{~nm}^{-1}$ at the DFT predicted Fermi energy, and the complexed series T2-T4 give a value of $1.2 \mathrm{~nm}^{-1}$, both in reasonable agreement with the experimental findings. The theoretically predicted conductance enhancement ratios upon TCNE complexation are slightly lower than the experimental value due to the calculated values for the isolated molecule being slightly higher than the measured ones, but the results are consistent with the experimental trend. The key aspect of the interpretation of the experimental results is that DFT predicts the position of these additional interference features always close ("pinned") to $E_{\mathrm{F}}$. The reason behind this lies in the strength of TCNE as electron acceptor, which forces the position of the part-filled orbital responsible for the Fano resonance automatically to adjust in order to achieve the required filling. This phenomenon also accounts for the relative independence of the interference feature energy on the location and orientation of the TCNE relative to the molecular backbone, which results in the well-defined "bumps" observed in the calculated conductance $v s$. energy curves, obtained as average of 500 different $T(\mathrm{E})$ curves. As the interference feature is reliably placed near $E_{\mathrm{F}}$, its contribution dominates charge transport and therefore the charge-transfer complex conductance is only a function of the room temperature fluctuations in the energy position of the zero-Kelvin Fano resonance (average room temperature $G(\mathrm{E})$ curves in Fig. $4 \mathrm{a}-\mathrm{c}$ ). Furthermore, as can be observed in Fig. 4d, there is significant spin splitting in the transmission curve, and this is again due to the partly-filled nature of the orbital associated with the molecule $\rightarrow$ TCNE charge transfer, behaving similarly to an unpaired electron.
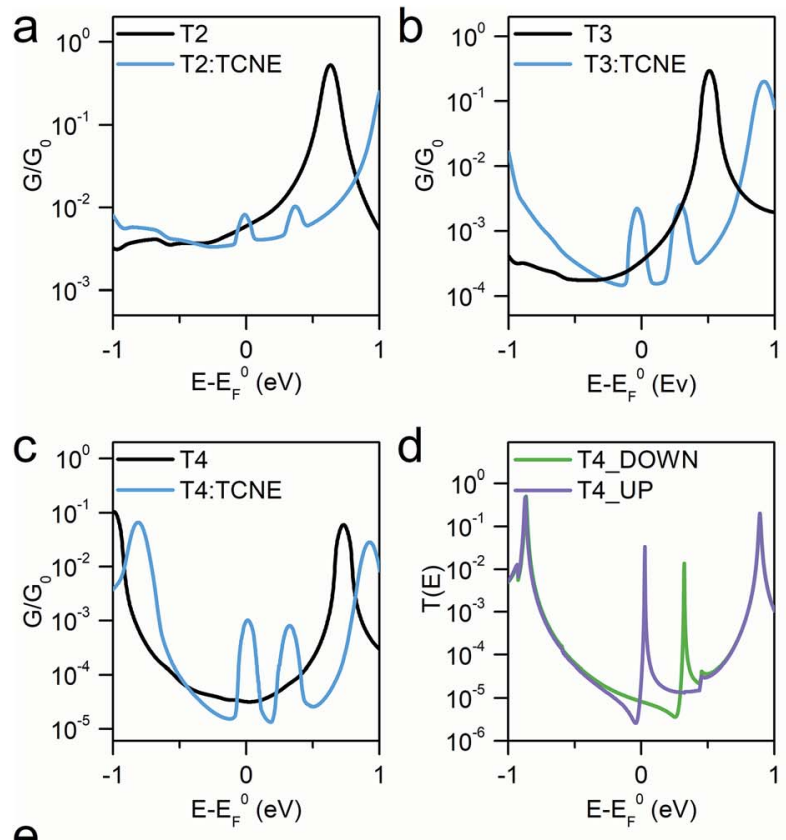

e

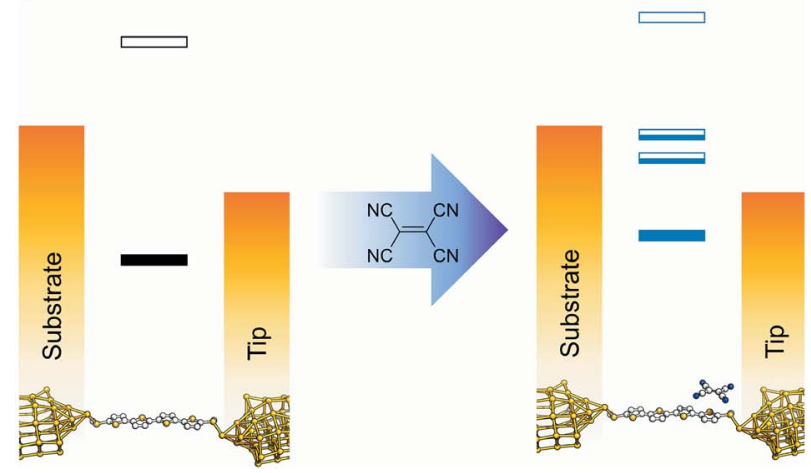

Fig. 4 Room temperature calculated conductance for the isolated molecules (black line) and complexed with TCNE (blue) for compounds T2 (a), T3 (b) and T4 (c), along with a representative spinpolarised $T(E)$ curve for the TCNE complex of T4 (d). Schematic diagram (e) showing the position of the DFT resonances for T4 respective to the two electrodes before and after TCNE complexation. The part-filled CT resonances in the HOMO-LUMO gap are responsible for the observed increase in conductance. 
Spin splitting results in the majority-spin contribution moving closer to $E_{\mathrm{F}}$ and the minority-spin contribution shifting away, and this phenomenon is responsible for the double bump in the calculated conductance curves (Fig. $4 \mathrm{a}-\mathrm{c}$ ). A similar behaviour was found in T2 and T3 (ESI, Fig. S5 $\dagger$ ). Therefore, the conductance at $E_{\mathrm{F}}$ is predicted to be different for the two spins, which could result in magnetoresistance effects.

As the CT resonance pinned to electrode $E_{\mathrm{F}}$ dominates charge transport through these molecular wires, one can postulate that such effect would not be affected even when changing the molecule-electrode contacts of the junction. As further evidence for the proposed mechanism of pinning of the CT transport resonance, we fabricated and characterised two additional $\alpha$-terthienyl compounds bearing thiol contacts. The same conductance measurements were carried out for these molecules. The results again showed conductance pinned at $10^{-3} G_{0}$ when complexed with TCNE (see ESI, Fig. S1 $\dagger$ ) even though their uncomplexed state gave completely different conductance values (ranging from $10^{-5} G_{0}$ to $10^{-4} G_{0}$ ). These additional results further support the proposed mechanism, with the conductance of molecule:TCNE complex confirmed independent of the contacts to the electrodes. The overall experimental and computational results confirm that (I) the energetic position of the charge-transfer interference feature is pinned at the Fermi level of the metallic electrodes and (II) this interference pinning phenomenon greatly reduces the attenuation coefficient $\beta$, thus enabling efficient charge transport even through relatively long molecular wires.

\section{Conclusions}

In summary, we have shown here that charge-transfer complexation of a series of oligothiophene molecular wires with TCNE leads to an up to $\sim 70$-fold increase in molecular junction conductance. The resulting complexes have conductance values nearly independent of the nature of the molecular backbone and contacts to the electrode and show a greatly reduced attenuation coefficient $\beta$ of $1.7 \mathrm{~nm}^{-1}$, enabling longrange efficient tunnelling. DFT calculations show that this phenomenon arises from constructive quantum interference features in the transmission profile (Fano resonances) pinned to the electrode Fermi level, which arise upon complexation. The conductance boost of more than one order of magnitude observed in the longer molecular wires, such as $\alpha$-quaterthiophene, not only offers a straightforward way to greatly enhance electrical conductance, but also makes these compounds and the CT complexation phenomenon interesting for the development of sensors with single-molecule sensitivity. Furthermore, a sharp transmission feature near the electrode Fermi level is a long-anticipated feature for the development of molecular thermoelectric devices with high Seebeck coefficient and figure of merit. Overall, this approach charts a clear path for the fabrication of molecular devices with some of the most desirable features, such as high conductance and long-range transport, with a high predicted efficiency in energy conversion. This study further stresses the importance of interference features in the transmission profile and the unique properties of nanoscale junctions operating in the quantum realm, where even weak supramolecular interactions can have a dramatic effect on junction behaviour.

\section{Methods}

\section{Synthesis}

T3 was prepared from $2,2^{\prime}: 5^{\prime}, 2^{\prime \prime}$-terthiophene by treatment with two equivalents of $n$-butyllithium, followed by quench with dimethyl disulfide. T2 was prepared from 2,2'-bithiophene by treatment with two equivalents of $n$-butyllithium, followed by quench with $\mathrm{S}_{8} / \mathrm{MeI}$. T4 was prepared by Stille coupling of $5,5^{\prime}$ dibromo-2,2'-bithiophene and trimethyl(5-(methylthio) thiophen-2-yl)stannane. Synthetic procedures and NMR/ HRMS/CNH data are provided in the ESI. $\dagger$

\section{Characterisation}

UV/vis spectra were recorded in $\mathrm{CH}_{2} \mathrm{Cl}_{2}$ on a double beam PerkinElmer $\lambda 25$ spectrometer, using a $1 \mathrm{~cm}$ quartz cell at room temperature.

\section{Conductance measurements}

The conductance of molecular junctions were determined using the STM break junction (STM-BJ) method. ${ }^{59}$ Au substrates were prepared by evaporating $\sim 100 \mathrm{~nm}$ of gold onto freshly cleaved mica sheets using a thermal evaporator under a vacuum of $10^{-7}$ Torr. The gold beads for Au substrate deposition was purchased from Kurt J. Lesker Company (99.999\%), and mica sheets were purchased from Ted Pella, Inc. Freshly annealed Au surfaces were incubated in solutions of the desired molecular wire $\left(10^{-3} \mathrm{M}, \mathrm{CH}_{2} \mathrm{Cl}_{2}\right.$ : Ethanol $\left.1: 1\right)$ for 30 minutes to allow adsorption of a sub-monolayer of the molecular wires, and then rinsed with ethanol and dried with high purity nitrogen gas. To form the TCNE complexes, a TCNE solution $\left(10^{-2} \mathrm{M}\right.$ in $\left.\mathrm{CH}_{2} \mathrm{Cl}_{2}\right)$ was dropped onto the prepared sub-monolayer of sample molecules and incubated for $1 \mathrm{~h}$. Samples were then copiously rinsed with ethanol and dried with high purity nitrogen gas. Conductance measurements were carried out in air at room temperature $\left(\sim 20{ }^{\circ} \mathrm{C}\right)$, with a bias of $100 \mathrm{mV}$ applied to the substrate (tip grounded). Conductance data was collected by driving a freshly cut Au tip into a freshly annealed Au substrate (35 $\mathrm{nm} \mathrm{s}^{-1}$ ) and then withdrawing it at constant speed $\left(20 \mathrm{~nm} \mathrm{~s}^{-1}\right)$. As the tip was pushed into the surface and then retracted, a fresh $\mathrm{Au}-\mathrm{Au}$ junction was formed, thinned down to a single atom (point contact), and finally broken upon further retraction. After the rupture of the junction, a molecule can bridge the tip-substrate gap. The tip was engaged towards the $\mathrm{Au}$ substrate until the tip-substrate current was $25 \mu \mathrm{A}$, and then crashed into the substrate a further $0.5 \mathrm{~nm}$ to ensure good physical contact between the tip and substrate before being withdrawn. This setting guaranteed that a clear $G_{0}$ point contact plateau is present in each trace. The current $(I)$ was recorded at a fixed tip-substrate bias $(V)$ and conductance $G$ is determined by Ohm's law $(G=I / V)$. The process was repeated thousands of times and the resulting current $v s$. distance traces were used for the construction of conductance histograms. 


\section{Theoretical conductance calculations}

The optimised geometry of each molecule and TCNE was calculated using the density functional code SIESTA. ${ }^{65}$ A double$\zeta$ basis set was used along with norm-conserving pseudopotentials. The energy cut-off to determine the fineness of the real space grid was 150 Rydbergs and the exchange correlation was described by GGA. ${ }^{66}$ The molecules were then relaxed until all forces were less than $0.01 \mathrm{eV} \AA^{-1}$. The binding of the molecules to gold electrodes was then calculated by a further geometry optimisation. A gold electrode consisting of a pyramid of 4 gold atoms attached to 8 layers of $\mathrm{Au}(111)$ each containing 9 atoms was constructed and the molecule attached through the anchor group, in the case of the thioacetate-protected molecules the acetyl group attached to the sulfur was removed. The resulting binding geometries show the binding distances between the terminal $\mathrm{Au}$ atom and the $\mathrm{S}$ atom of the anchor group to be $2.5 \AA$ (T2-T4) and the Au-S-C angles to be $120^{\circ}$ (T2-T4). To model the addition of TCNE molecules to the junction we calculate the binding energy due to the formation of a charge transfer complex in each of the three molecules. Firstly, the optimum binding location is found by moving the TCNE across the backbone and locating the binding energy minimum. This geometry was then used to perform a geometry relaxation to give the optimised geometry of the complex. The shape and position of the Fano resonance is dependent on the binding geometry between the TCNE and molecule so, to account for geometry fluctuations following the previous theoretical model, ${ }^{17} 500$ geometries of the TCNE molecule are taken in the vicinity of the optimum position. The junction geometries are then utilised in the DFT code SIESTA to generate a Hamiltonian. The extended molecule in each case is constructed with the gold electrodes incorporating 8 layers of $\mathrm{Au}(111)$ with each layer containing 9 atoms, and the quantum transport code GOL$\mathrm{LUM}^{67}$ was then used to calculate the spin-polarised zero-bias transmission coefficient which is related to the electrical conductance through the Landauer formalism. We then calculate the conductance at room temperature by evaluating:

$$
G\left(E_{\mathrm{F}}\right)=\frac{2 e^{2}}{h} \int T(\mathrm{E})\left(-\frac{\mathrm{d} f}{\mathrm{~d} E}\right) \mathrm{d} E
$$

where $f$ is the Fermi distribution. For the complexed molecules an average is taken over all calculated geometries.

\section{Data availability}

Proton and ${ }^{13} \mathrm{C}$ NMR spectra in Bruker data format for the compounds used in this study are deposited in the University of Liverpool data catalogue (collection \#464) at the address http:// datacat.liverpool.ac.uk/464/ and at DOI: 10.17638/ datacat.liverpool.ac.uk/464.

Single-molecule conductance and DFT data is available from the authors upon request.

\section{Conflicts of interest}

There are no conflicts to declare.

\section{Acknowledgements}

This work was supported by UK EPSRC under grants EP/ H035818/1 (Medium Effects in Single-Molecule Electronics, Lancaster), EP/H035184/1 (Medium Effects in Single-Molecule Electronics, Liverpool) EP/M005046/1 (Single-Molecule PhotoSpintronics, Liverpool) and by US NSF grants ECCS 1609788 and ECCS 1231967 (Athens).

\section{References}

1 E. D. Fung, O. Adak, G. Lovat, D. Scarabelli and L. Venkataraman, Nano Lett., 2017, 17, 1255-1261.

2 K. Yoshida, K. Shibata and K. Hirakawa, Phys. Rev. Lett., 2015, 115, 1-5.

3 J. Zhou, K. Wang, B. Xu and Y. Dubi, J. Am. Chem. Soc., 2018, 140, 70-73.

4 H. M. Osorio, S. Catarelli, P. Cea, J. B. G. Gluyas, F. Hartl, S. J. Higgins, E. Leary, P. J. Low, S. Martín, R. J. Nichols, J. Tory, J. Ulstrup, A. Vezzoli, D. C. Milan and Q. Zeng, J. Am. Chem. Soc., 2015, 137, 14319-14328.

5 Z. Li, I. Pobelov, B. Han, T. Wandlowski, A. Błaszczyk and M. Mayor, Nanotechnology, 2007, 18, 044018.

6 M. Baghernejad, D. Z. Manrique, C. Li, T. Pope, U. Zhumaev, I. Pobelov, P. Moreno-García, V. Kaliginedi, C. Huang, W. Hong, C. Lambert and T. Wandlowski, Chem. Commun., 2014, 50, 15975-15978.

7 R. J. Brooke, C. Jin, D. S. Szumski, R. J. Nichols, B. Mao, K. S. Thygesen and W. Schwarzacher, Nano Lett., 2015, 15, 275-280.

8 Y. Kim, W. Jeong, K. Kim, W. Lee and P. Reddy, Nat. Nanotechnol., 2014, 9, 881-885.

9 D. Xiang, H. Jeong, D. Kim, T. Lee, Y. Cheng, Q. Wang and D. Mayer, Nano Lett., 2013, 13, 2809-2813.

10 J. Park, A. N. Pasupathy, J. I. Goldsmith, C. Chang, Y. Yaish, J. R. Petta, M. Rinkoski, J. P. Sethna, H. D. Abruña, P. L. McEuen and D. C. Ralph, Nature, 2002, 417, 722-725.

11 A. C. Aragonès, N. L. Haworth, N. Darwish, S. Ciampi, N. J. Bloomfield, G. G. Wallace, I. Diez-Perez and M. L. Coote, Nature, 2016, 531, 88-91.

12 V. Fatemi, M. Kamenetska, J. B. Neaton and L. Venkataraman, Nano Lett., 2011, 11, 1988-1992.

13 E. Leary, H. Höbenreich, S. J. Higgins, H. van Zalinge, W. Haiss, R. J. Nichols, C. Finch, I. Grace, C. J. Lambert, R. McGrath and J. Smerdon, Phys. Rev. Lett., 2009, 102, 086801.

14 B. Choi, B. Capozzi, S. Ahn, A. Turkiewicz, G. Lovat, C. Nuckolls, M. L. Steigerwald, L. Venkataraman and X. Roy, Chem. Sci., 2016, 5, 476-482.

15 D. C. Milan, O. A. Al-Owaedi, M.-C. Oerthel, S. MarquésGonzález, R. J. Brooke, M. R. Bryce, P. Cea, J. Ferrer, S. J. Higgins, C. J. Lambert, P. J. Low, D. Z. Manrique, S. Martin, R. J. Nichols, W. Schwarzacher and V. M. GarcíaSuárez, J. Phys. Chem. C, 2016, 120, 15666-15674.

16 S. Nakashima, Y. Takahashi and M. Kiguchi, Beilstein J. Nanotechnol., 2011, 2, 755-759. 
17 A. Vezzoli, I. Grace, C. Brooke, K. Wang, C. J. Lambert, B. Xu, R. J. Nichols and S. J. Higgins, Nanoscale, 2015, 7, 1894918955.

18 R. García, M. Á. Herranz, E. Leary, M. T. González, G. R. Bollinger, M. Bürkle, L. A. Zotti, Y. Asai, F. Pauly, J. C. Cuevas, N. Agraït and N. Martín, Beilstein J. Org. Chem., 2015, 11, 1068-1078.

19 B. S. T. Kasibhatla, A. P. Labonté, F. Zahid, R. G. Reifenberger, S. Datta and C. P. Kubiak, J. Phys. Chem. B, 2003, 107, 12378-12382.

20 J. Del Re, M. H. Moore, B. R. Ratna and A. S. Blum, Phys. Chem. Chem. Phys., 2013, 15, 8318-8323.

21 W. Zhang, S. Gan, A. Vezzoli, R. J. Davidson, D. C. Milan, K. V. Luzyanin, S. J. Higgins, R. J. Nichols, A. Beeby, P. J. Low, B. Li and L. Niu, ACS Nano, 2016, 10, 5212-5220.

22 A. Vezzoli, I. M. Grace, C. Brooke, R. J. Nichols, C. J. Lambert and S. J. Higgins, J. Chem. Phys., 2017, 146, 092307.

23 M. Kiguchi, S. Nakashima, T. Tada, S. Watanabe, S. Tsuda, Y. Tsuji and J. Terao, Small, 2012, 8, 726-730.

24 T. Nishino, T. Ito and Y. Umezawa, Proc. Natl. Acad. Sci. U. S. A., 2005, 102, 5659-5662.

25 S. Martín, I. Grace, M. R. Bryce, C. Wang, R. Jitchati, A. S. Batsanov, S. J. Higgins, C. J. Lambert and R. J. Nichols, J. Am. Chem. Soc., 2010, 132, 9157-9164.

26 S. Wu, M. T. González, R. Huber, S. Grunder, M. Mayor, C. Schönenberger and M. Calame, Nat. Nanotechnol., 2008, 3, 569-574.

27 S. T. Schneebeli, M. Kamenetska, Z. Cheng, R. Skouta, R. A. Friesner, L. Venkataraman and R. Breslow, J. Chem. Soc., Chem. Commun., 2011, 133, 2136-2139.

28 T. Ghane, D. Nozaki, A. Dianat, A. Vladyka, R. Gutierrez, J. P. Chinta, S. Yitzchaik, M. Calame and G. Cuniberti, J. Phys. Chem. C, 2015, 119, 6344-6355.

29 R. Frisenda, V. A. E. C. Janssen, F. C. Grozema, H. S. J. van der Zant and N. Renaud, Nat. Chem., 2016, 8, 1099-1104.

30 S. Fujii, T. Tada, Y. Komoto, T. Osuga, T. Murase, M. Fujita and M. Kiguchi, J. Am. Chem. Soc., 2015, 137, 5939-5947.

31 C. Guo, K. Wang, E. Zerah-Harush, J. Hamill, B. Wang, Y. Dubi and B. Xu, Nat. Chem., 2016, 8, 484-490.

32 T. Nishino, N. Hayashi and P. T. Bui, J. Am. Chem. Soc., 2013, 135, 4592-4595.

33 S. Chang, J. He, A. Kibel, M. Lee, O. Sankey, P. Zhang and S. Lindsay, Nat. Nanotechnol., 2009, 4, 297-301.

34 T. Nishino, H. Shiigi, M. Kiguchi and T. Nagaoka, Chem. Commun., 2017, 53, 5212-5215.

35 P. Sautet and C. Joachim, Chem. Phys. Lett., 1988, 153, 511516.

36 C. J. Lambert, Chem. Soc. Rev., 2015, 44, 875-888.

37 G. Yang, S. Sangtarash, Z. Liu, X. Li, H. Sadeghi, Z. Tan, R. Li, J. Zheng, X. Dong, J.-Y. Liu, Y. Yang, J. Shi, Z. Xiao, G. Zhang, C. Lambert, W. Hong and D. Zhang, Chem. Sci., 2017, 8, 7505-7509.

38 M. Gantenbein, L. Wang, A. A. Al-jobory, A. K. Ismael, C. J. Lambert, W. Hong and M. R. Bryce, Sci. Rep., 2017, 7, 1794.

39 C. R. Arroyo, S. Tarkuc, R. Frisenda, J. S. Seldenthuis, C. H. M. Woerde, R. Eelkema, F. C. Grozema and
H. S. J. van der Zant, Angew. Chem., Int. Ed., 2013, 52, 3152-3155.

40 G. C. Solomon, D. Q. Andrews, R. P. Van Duyne and M. A. Ratner, ChemPhysChem, 2009, 10, 257-264.

41 H. Valkenier, C. M. Guédon, T. Markussen, K. S. Thygesen, S. J. van der Molen and J. C. Hummelen, Phys. Chem. Chem. Phys., 2014, 16, 653-662.

42 G. C. Solomon, D. Q. Andrews, R. H. Goldsmith, T. Hansen, M. R. Wasielewski, R. P. Van Duyne and M. A. Ratner, J. Am. Chem. Soc., 2008, 130, 17301-17308.

43 M. H. Garner, H. Li, Y. Chen, T. A. Su, Z. Shangguan, D. W. Paley, T. Liu, F. Ng, H. Li, S. Xiao, C. Nuckolls, L. Venkataraman and G. C. Solomon, Nature, 2018, 558, 416-419.

44 R. Miao, H. Xu, M. Skripnik, L. Cui, K. Wang, K. G. L. Pedersen, M. Leijnse, F. Pauly, K. Wärnmark, E. Meyhofer, P. Reddy and H. Linke, Nano Lett., 2018, 18, 5666-5672.

45 G. Breit and E. Wigner, Phys. Rev., 1936, 49, 519-531.

46 C. J. Lambert and M. F. Thorpe, Phys. Rev. B: Condens. Matter Mater. Phys., 1983, 27, 715-726.

47 M. Ya. Azbel and P. Soven, Phys. Rev. B: Condens. Matter Mater. Phys., 1983, 27, 831-835.

48 C. J. Lambert, J. Phys. C: Solid State Phys., 1984, 17, 24012414.

49 Y. Gefen, Y. Imry and M. Y. Azbel, Phys. Rev. Lett., 1984, 52, 129-132.

50 M. Büttiker, Y. Imry, R. Landauer and S. Pinhas, Phys. Rev. B: Condens. Matter Mater. Phys., 1985, 31, 6207-6215.

51 M. Magoga and C. Joachim, Phys. Rev. B: Condens. Matter Mater. Phys., 1999, 59, 16011-16021.

52 H. Vazquez, R. Skouta, S. Schneebeli, M. Kamenetska, R. Breslow, L. Venkataraman and M. S. Hybertsen, Nat. Nanotechnol., 2012, 7, 663-667.

53 A. Borges, J. Xia, S. H. Liu, L. Venkataraman and G. C. Solomon, Nano Lett., 2017, 17, 4436-4442.

54 J. Ferraris, D. O. Cowan, V. Walatka and J. H. Perlstein, J. Am. Chem. Soc., 1973, 95, 948-949.

55 D. Jerome, A. Mazaud, M. Ribault and K. Bechgaard, J. Phys., Lett., 1980, 41, 95-98.

56 J. E. Frey, T. Aiello, D. N. Beaman, H. Hutson, S. R. Lang and J. J. Puckett, J. Org. Chem., 1995, 60, 2891-2901.

57 Z. Yoshida and T. Kobayashi, Tetrahedron, 1970, 26, 267-271. 58 R. Abu-Eittah and F. Al-Sugeir, Can. J. Chem., 1976, 54, 37053712 .

59 B. Xu and N. Tao, Science, 2003, 301, 1221-1223.

60 B. Capozzi, E. J. Dell, T. C. Berkelbach, D. R. Reichman, L. Venkataraman and L. M. Campos, J. Am. Chem. Soc., 2014, 136, 10486-10492.

61 H. Atesci, V. Kaliginedi, J. A. Celis Gil, H. Ozawa, J. M. Thijssen, P. Broekmann, M. Haga and S. J. van der Molen, Nat. Nanotechnol., 2018, 13, 117-121.

62 B. Xu, D. Fichou, G. Horowitz and F. Garnier, Synth. Met., 1991, 42, 2319-2322.

63 S. Hotta and H. Kobayashi, Synth. Met., 1994, 66, 117-122.

64 T. Papadopoulos, I. Grace and C. Lambert, Phys. Rev. B: Condens. Matter Mater. Phys., 2006, 74, 193306. 
65 J. M. Soler, E. Artacho, J. D. Gale, A. García, J. Junquera, P. Ordejón and D. Sánchez-Portal, J. Phys.: Condens. Matter, 2002, 14, 2745-2779.

66 J. P. Perdew, K. Burke and M. Ernzerhof, Phys. Rev. Lett., 1996, 77, 3865-3868.
67 J. Ferrer, C. J. Lambert, V. M. García-Suárez, D. Z. Manrique, D. Visontai, L. Oroszlany, R. Rodríguez-Ferradás, I. Grace, S. W. D. Bailey, K. Gillemot, H. Sadeghi and L. A. Algharagholy, New J. Phys., 2014, 16, 093029. 